\title{
Empirical Correlations between Cumulative Absolute Velocity and Amplitude-Based Ground Motion Intensity Measures
}

\author{
Brendon A Bradley a) b)
}

Empirical correlation equations are developed between cumulative absolute velocity $(C A V)$ and other common ground motion intensity measures, namely, peak ground acceleration (PGA), peak ground velocity (PGV), 5\% damped pseudo spectral acceleration (SA), acceleration spectrum intensity (ASI), spectrum intensity (SI), and displacement spectrum intensity (DSI). It is found that, for a given earthquake rupture, $C A V$ has the strongest correlation with high and moderate frequency intensity measures (IMs), i.e. ASI, PGA, PGV and highfrequency $S A$, and to a lesser extent with low frequency IMs (DSI and lowfrequency $S A$ ). The largest positive correlations of approximately 0.7 however are not high in an absolute sense, a result of the cumulative nature of $C A V$. The equations allow estimation of the joint distribution of these intensity measures for a given earthquake rupture, enabling the inclusion of $C A V$, and its benefit as a cumulative intensity measure, in seismic hazard analysis, ground motion selection, and seismic response analysis.

\section{INTRODUCTION}

Cumulative absolute velocity is defined as the integral of the absolute value of the acceleration time history of a ground motion (EPRI 1988), specifically:

$$
C A V=\int_{0}^{t_{\max }}|a(t)| d t
$$

where $|a(t)|$ is the absolute value of the acceleration of the ground motion at time $t$, and $t_{\text {max }}$ is the total duration of ground motion shaking. It is clearly evident from its definition that $C A V$ increases with time. Therefore $C A V$ includes cumulative effects of ground motion shaking, which are not captured by many amplitude-based ground motion intensity measures

\footnotetext{
a) Department of Civil and Natural Resources Engineering, University of Canterbury, Christchurch, New Zealand

b) Institute of Science and Engineering, Chuo University, Tokyo, Japan
} 
such as peak ground acceleration, PGA and peak ground velocity, PGV.

As a result of its cumulative nature, EPRI (1988) used CAV as a parameter to assess the exceedance of the operating basis earthquake (OBE) at nuclear power plant sites, in addition to response spectral ordinates, which on their own were seen as an insufficient descriptor of the severity of small magnitude-induced ground motions. EPRI (2006) subsequently modified the use of $C A V$ in the seismic assessment of nuclear power plants by using a standardized version of $C A V, C A V_{S T D}$, to filter out small magnitude events in probabilistic seismic hazard calculations. Koliopoulos et al. (1998) also found a strong correlation between $C A V$ and spectrum intensity, SI (Koliopoulos et al. 1998). For performance-based earthquake engineering calculations, $C A V$ has been used as an intensity measure (IM) for scaling ground motion records in several studies (Bradley et al. 2009a, Kramer and Mitchell 2006). Kramer and Mitchell (2006) also found that a slightly modified version of $C A V, C A V_{5}$ exhibited strong correlations with the build up of excess pore water pressures in soils and the occurrence of liquefaction. Bradley et al. (2009a) found that $C A V$ was a relatively good predictor of the peak displacement of pile-founded structures in liquefiable soils. While the aforementioned literature illustrates that there are several variants of $C A V$, only the original definition (i.e. EPRI (1988)) is considered herein.

Despite the above findings, it must still be remembered that $C A V$ is a highly simplified, and therefore in many cases, incomplete description of a ground motions severity on a particular structure and its contents. To clearly demonstrate this, consider two different scenarios with causal magnitudes of 6.0 and 7.5; and source-to-site distances of 10 and $70 \mathrm{~km}$, respectively. These scenarios are of interest because the model of Campbell and Bozorgnia (2010) predicts the same median and standard deviation of $C A V$ for these two cases (a median of 0.30 g-s for a vertical surface-rupturing strike-slip fault and rock site). Figure 1 illustrates the time histories and response spectra of two ground motions with magnitude and source-tosite distance similar to these identified scenarios. It can be seen that while both ground motions have $C A V$ values similar to the median of $0.3 \mathrm{~g}-\mathrm{s}$, the time histories of the motions are completely different with the small magnitude near-source event having a greater highfrequency intensity, smaller low-frequency intensity, and shorter duration than the larger magnitude event. While these two motions may lead to similar levels of seismic demand in particular cases, they may also result in significantly different demands in others.

In general, it should be clear that a precise estimate of the severity of a ground motion for a particular system requires a combination of the cumulative nature and aforementioned 
benefits of $C A V$, with other IMs which account for the amplitude and duration characteristics of a ground motion. Ground motion selection using the generalized conditional intensity measure (GCIM) approach (Bradley 2010b) provides a probabilistic framework in which any number of ground motion IMs can be considered in the selection of ground motions for seismic response analysis. A key component of the GCIM framework is the availability of equations which provide the correlation between (the logarithm of) various ground motion intensity measures for a given earthquake rupture. This manuscript develops such empirical correlation equations between $C A V$ and several other IMs. Firstly, the considered IMs, ground motion prediction equations (GMPEs), empirical ground motion dataset, and correlation methodology are presented. Secondly, the observed correlation results are discussed and parametric models developed. Finally, the use of correlation coefficients in enabling $C A V$ to be used in ground motion selection is demonstrated.

\section{EMPIRICAL CORRELATION OF CAV WITH OTHER INTENSITY MEASURES}

\section{INTENSITY MEASURES EXAMINED}

The specific intensity measures considered herein for correlations with $C A V$ are: (i) peak ground acceleration, PGA; (ii) peak ground velocity, $P G V$; (iii) 5\% damped pseudo spectral acceleration, $S A$, for periods from 0.01 to 10 seconds (herein referred to as spectral acceleration for brevity); (iv) acceleration spectrum intensity, ASI (Von Thun et al. 1988); (v) spectrum intensity, SI (Housner 1952); and (vi) displacement spectrum intensity, DSI (Bradley 2010a). The number of ground motion IMs considered herein is limited because of scope and is not intended to imply that other IMs are not important, or that the considered IMs are best used in combination with $C A V$ in seismic hazard analysis, seismic response analysis, or ground motion selection.

\section{GROUND MOTION PREDICTION EQUATIONS ADOPTED}

The predicted distributions of the aforementioned intensity measures for a given (active shallow crustal) rupture scenario were obtained using various ground motion prediction equations (GMPEs). Distributions of PGA, PGV, SA, ASI, SI, and DSI were computed using four of the NGA (Power et al. 2008) ground motion prediction equations: Boore and Atkinson (2008), Chiou and Youngs (2008), Campbell and Bozorgnia (2008), and Abrahamson and Silva (2008). These four GMPEs are herein referred to as BA08, CY08, CB08 and AS08, respectively. The four NGA GMPEs provide explicit predictions for PGA, PGV and SA. These GMPEs can also be used to predict ASI, SI and DSI using analytical equations based on 
SA GMPEs (Bradley 2009, Bradley 2010a, Bradley et al. 2009b). Herein, for example, a prediction of SI using the Bradley et al. (2009b) analytical equation and the BA08 SA model is simply referred to as a BA08 SI prediction.

Unlike the aforementioned prediction equations used to compute PGA, SA etc. which build upon decades of research into GMPEs for these IMs, $C A V$ is a relatively new IM which has not been used widely in seismic hazard analysis, and therefore few GMPEs for its prediction exist. Campbell and Bozorgnia (2010) (CB10), Danciu and Tselentis (2007) (DT07) provide the only prediction equations, that the author is aware of, for $C A V$. The DT07 GMPE was developed based on 355 ground motions from 151 earthquakes in Greece. Because of a lack of ground motions, DT07 decided to include many recordings from instruments located in buildings (up to the second floor), as well as free-field sites. Furthermore, because of a lack of finite fault models for Greek earthquakes, DT07 also use epicentral distance rather than finite fault-based distance metrics. The CB10 GMPE, on the other hand uses the same ground motion dataset used in developing the CB08 NGA GMPE which contains 1561 ground motions from 64 earthquakes (Campbell and Bozorgnia 2010). Campbell and Bozorgnia (2010) also compared their GMPE with that of DT07, noting that while the models were similar for small and moderate magnitude events (i.e. $M_{w}<6$ ), a lack of magnitude saturation in the DT07 GMPE lead to significant difference for larger magnitudes. The omission of magnitude saturation in the DT07 model is presumably a result of the paucity of records from large magnitude events in Greece, with only 5 motions from 3 earthquakes with $M_{w}>6.5$ (Danciu and Tselentis 2007).

An aim of the empirical correlation equations developed in this manuscript is that they be generally applicable for ground motions resulting from active shallow crustal earthquakes. For PGA and SA's, in particular, the NGA GMPEs have been demonstrated to be widely applicable for active shallow crustal tectonic regions throughout the world (Peruš and Fajfar 2010, Scasserra et al. 2009, Shoja-Taheri et al. 2010, e.g. Stafford et al. 2008). While no such formal analyses have shown the same to be the case for PGV and the CB10 CAV model, Campbell and Bozorgnia (2010) suggest that the CB10 model will also be generally applicable for active shallow crustal tectonic regions worldwide (presumably on the basis that it uses the same ground motion dataset as the CB10 NGA model). Conversely, on the basis of the comparison shown by Campbell and Bozorgnia (2010), it can be concluded that the DT07 GMPE is not applicable for ground motions from large magnitude earthquakes in active shallow crustal tectonic regions, which typically dominate seismic hazard analyses. As a 
result, only the CB10 GMPE is considered herein to estimate CAV for the subsequent correlation analyses. The fact that only a single GMPE is used for $C A V$, while multiple are used for the other IMs, gives rise to the possibility of an under-estimation of epistemic uncertainty in the computed correlations with $C A V$. Such a possibility is further discussed in regard to the obtained results from the correlation analyses later in the manuscript.

\section{GROUND MOTION DATABASE}

Ground motion records from active shallow crustal earthquakes in the NGA database (Chiou et al. 2008) (http://peer.berkeley.edu/nga) were used to obtain empirical values of $C A V, P G A, P G V, S A, A S I, S I$ and DSI for use in the subsequent correlation analyses. The specific subset of the NGA database used is that of Chiou and Youngs (2006), but with the removal of the majority of (small amplitude) ground motions from aftershocks of the Chi-Chi and Northridge earthquakes (as several of the considered GMPEs do not distinguish between mainshock and aftershock ground motions). The magnitude, and source-to-site distance distribution of the resulting dataset of 1842 ground motions is given in Figure 2. While this dataset is not the same as the individual subsets used by the various GMPEs developed from the NGA database, all of these GMPEs are applicable for a range of predictor variables (i.e. magnitude, distance, etc.) which encompass all of those records used in the present study. This ensures that the point estimated correlation coefficients computed subsequently are unbiased. For example, Figure 3 illustrates the distributions of the $C A V$ (normalized) interand intra-event residuals from the ground motions in the dataset in comparison with the standard normal distribution. The fact that both empirical inter- and intra-event distributions lie within the Kolmorogov-Smirnov goodness-of-fit bounds (at the 5\% significance level) illustrates the applicability of the CB10 CAV model for this dataset (similar observations were obtained for the other considered IMs). Empirical IM values and the predicted IM distribution for each record were only computed if the IM was within the usable period range of the record. For example, if the maximum usable period was 2.0 seconds then no spectral ordinates above 2.0 seconds would be computed. SI and DSI were therefore also only computed if the maximum useable period was greater than or equal to 2.5 and 5.0 seconds, respectively. Because $P G V$ is also affected by the high pass filter cut-off period (Akkar and Bommer 2007) then those motions with cut-off periods less than 4.0 seconds were also not considered in correlation analyses involving $P G V$.

\section{METHODOLOGY FOR CORRELATION COEFFICIENT COMPUTATION}

Prediction equations for ground motion IMs have the general form: 


$$
\operatorname{lnI} M_{i}=f_{i}\left(\text { Rup }_{k}, \text { Site }\right)+\varepsilon_{T_{i}} \sigma_{T_{i}}(\text { Rup } \text { R }, \text { Site })
$$

where $\ln$ is the natural logarithm; $f_{i}\left(\right.$ Rup $_{k}$, Site $)=\mu_{\text {InIM }_{i} \mid R u p_{k}}$ and $\sigma_{T_{i}}\left(\right.$ Rup $_{k}$, Site $)=$ $\sigma_{T_{\text {InIM }} \mid R_{\text {Rup }}}$ are the predicted mean and standard deviation of $\operatorname{lnI} M_{i}$, which are a function of the earthquake rupture and site of interest (e.g. magnitude, source-to-site distance etc.); and $\varepsilon_{T_{i}}$ is the (normalized) total residual for $I M_{i}$ (assumed to have a standard normal distribution).

By rearranging Equation (2), the total residual for a particular ground motion $n, \varepsilon_{T_{i, n}}$, can be interpreted as the number of standard deviations the ground motion has $\ln I M_{i}$ above the predicted mean:

$$
\varepsilon_{T_{i, n}}=\frac{\operatorname{lnIM}_{i, n}-\mu_{\operatorname{lnIM}_{i} \mid R u p_{k}}}{\sigma_{T_{\text {lnIM }} \mid \text { Rup }}}
$$

From the linear relationship between $\ln I M_{i, n}$ and $\varepsilon_{T_{i, n}}$ in Equation (3) it follows that the correlation between two IMs, for a given earthquake rupture, is equal to the correlation between the normalized residuals, i.e. $\rho_{\operatorname{lnIM}_{i}\left|R u p_{k}, \operatorname{lnI} I M_{j}\right| R u p_{k}}=\rho_{\varepsilon_{T_{i}}, \varepsilon_{T_{j}}}$. Herein, for brevity, this correlation between two intensity measures, $\rho_{\operatorname{lnIM}_{i}\left|R u p_{k}, \operatorname{lnIM}_{j}\right| R u p_{k}}$, will simply be referred to as $\rho_{\text {InIM }_{i}, I n I M_{j}}$ (where the conditioning on $R u p_{k}$ and the same location is implied).

The values of $\varepsilon_{T_{i, n}}$ obtained for all of the ground motions in the dataset are correlated because of the use of multiple records from a single event. In order to account for this correlation the total residual can be expressed as the sum of an inter- and intra-event residual. The relationship between the total (normalized) residual, $\varepsilon_{T_{i}}$, and the normalized inter- and intra-event residuals is given by:

$$
\varepsilon_{T_{i}} \sigma_{T_{i}}=\eta_{i} \tau_{i}+\varepsilon_{i} \sigma_{i}
$$

where $\eta_{i}$ and $\varepsilon_{i}$ are the (normalized) inter- and intra-event residuals; and $\tau_{i}$ and $\sigma_{i}$ are the inter- and intra-event standard deviations (where the subscript of all terms in Equation (4) pertains to $\left.I M_{i}\right)$. The inter- and intra-event residuals were obtained by performed mixedeffects regression on the total residuals (e.g. Scasserra et al. (2009)). Other correlations, due to multiple recordings at a single location (Chen and Tsai 2002) and spatial correlation of ground motions (Jayaram and Baker 2010) were not considered in the GMPE's examined in this study and therefore are not discussed further herein.

As $\eta_{i}$ and $\varepsilon_{i}$ are assumed to be independent in the GMPEs used in this study (Abrahamson et al. 2008, Abrahamson and Youngs 1992), then it is possible to estimate the correlations 
between the inter- and intra-event residuals of the different IMs (assuming they are well approximated as bi-variate normal) using the Pearson-product-moment correlation estimator (Ang and Tang 2007):

$$
\rho_{x, y}=\frac{\sum_{n}[(x-\bar{x})(y-\bar{y})]}{\sqrt{\sum_{n}\left[(x-\bar{x})^{2}\right] \sum_{n}\left[(y-\bar{y})^{2}\right]}}
$$

where $x$ and $y$ are generic variables (i.e. in this case $\eta_{i}$ and $\eta_{j}$ for the inter-event correlation and $\varepsilon_{i}$ and $\varepsilon_{j}$ for the intra-event correlation), $\bar{x}$ and $\bar{y}$ are the sample means of $x$ and $y$; and $\sum_{n}$ [ ] represents summation over the number of ground motion records (i.e. $n=1-$ $N_{\text {record }}$ ), or the number of earthquakes (for computation of the inter-event correlation). Thus, Equation (5) can be used to estimate $\rho_{\eta_{i}, \eta_{j}}$ and $\rho_{\varepsilon_{i}, \varepsilon_{j}}$. Using the definition of the correlation coefficient, the correlation between the total residuals can then be found from the inter- and intra-event correlations by:

$$
\rho_{\varepsilon_{T_{i}}, \varepsilon_{T_{j}}}=\frac{1}{\sigma_{T_{i}} \sigma_{T_{j}}}\left[\rho_{\eta_{i}, \eta_{j}} \tau_{i} \tau_{j}+\rho_{\varepsilon_{i}, \varepsilon_{j}} \sigma_{i} \sigma_{j}\right]
$$

Bradley (2011) showed that, while strictly speaking the correlation between the total residuals is a function of the particular rupture scenario (as $\tau_{i}, \sigma_{i}$ etc. are potentially a function of rupture scenario), it is very insensitive to rupture scenario (because the ratio of the intraevent and total standard deviation is relatively constant), and hence can be computed using the mean ratio (for the ground motions used) between the intra-event and total standard deviations of a particular GMPE.

As can be seen from Equation (3), the total residual for a particular $n^{\text {th }}$ record, $\varepsilon_{i, n}$, is a function of the GMPE used to estimate the mean, $\mu_{\operatorname{lnIM} M_{i} \mid R u p_{k}}$, and standard deviation, $\sigma_{\text {InIM }_{i} \mid R u p_{k}}$ of the ground motion intensity measure. Therefore, by way of Equation (5), the estimated correlation coefficient is also a function of the GMPE used. The (epistemic) uncertainty in the correlation coefficient due to GMPE uncertainty is accounted by using the four aforementioned GMPE's developed for active shallow crustal tectonic regions. It is assumed that each GMPE is equally valid, meaning that each is given a logic tree weight of 0.25 (Kulkarni et al. 1984).

In addition to uncertainty due to the selected GMPEs, Equation (5) provides only the point-estimate of the correlation coefficient, which in reality contains (epistemic) uncertainty due to the finite number of recordings (i.e. $N_{\text {record }}$ ) used in its determination. In order to account for such finite sample uncertainty it is first beneficial to use the approximate variance 
stabilizing Fisher $\mathrm{z}$ transformation, which is defined by:

$$
z=\frac{1}{2} \ln \left(\frac{1+\rho}{1-\rho}\right)=\tanh ^{-1}(\rho)
$$

where $\rho$ is the pearson correlation coefficient, $\ln$ is the natural logarithm; $\tanh ^{-1}$ is the inverse hyperbolic tangent function; and $z$ is the transformed correlation coefficient. It can be shown that $z$ has approximately a normal distribution with mean given by Equation (7), and variance given by (Ang and Tang 2007):

$$
\operatorname{Var}[z]=\frac{1}{N-3}
$$

where $N$ is the sample size. An alternative to the use of Equation (8) for estimating the variance in $z$, which is utilized herein and also applicable for all sample sizes, is the bootstrap method (Ang and Tang 2007), in which bootstrap samples are created by random selecting data (pairs in this case) with replacement.

\section{COMPUTED EMPIRICAL CORRELATION}

\section{EMPIRICAL RESULTS}

Figure 4 illustrates an example of the normalised inter- and intra-event residuals for $C A V$ and PGA obtained from (a single bootstrap sample of) the adopted dataset using the BA08 PGA GMPE. It is important to note the larger number of data points for the intra-event residuals compared to the inter-event residuals which consequently leads to the intra-event correlations having less finite sample uncertainty than the inter-event correlations. For this example, the correlations between the inter- and intra-event residuals of $P G A$ and $C A V$ are 0.63 and 0.70 , respectively, while the correlation between total residuals computed via Equation (6) is 0.687.

Figure 5a- Figure 5e illustrate the correlation coefficients computed between $C A V$ and $P G A, P G V$, ASI, SI, and DSI, respectively. For each of the different $C A V-I M_{i}$ combinations, individual boxplots (Ang and Tang 2007) are used to illustrate the variation in the computed correlation coefficient (using a single GMPE) due to finite sample uncertainty (obtained from bootstrap sampling). The four different boxplots for each IM combination correspond to the four different GMPEs used to compute the median and standard deviation of $I M_{i}$ (recall that only one GMPE was used for $C A V$ ). It can be seen that the correlations between $C A V$ and the high-frequency IMs, PGA, and ASI are the largest (approximately 0.7) closely followed by $P G V$ and the moderate-frequency SI (approximately 0.68-0.69). The correlation between 
$C A V$ and DSI is the lowest of the considered IM combinations with a median value in the order of 0.55 .

It can be seen that variability in the correlation coefficients (as indicated by the 'whiskers' of the box plots) due to sample uncertainty is approximately 0.07-0.09 for $I M_{i}$ 's $P G A, P G V$, $A S I$, and SI; and approximately 0.11-0.14 for DSI. The difference in the sample size uncertainty in the correlation between $C A V$ and $D S I$ illustrates the non-constant variance of the sample size uncertainty as a function of the correlation coefficient (and hence why Equation (7) is later considered). It can also be seen that while there is some difference between the correlation coefficients estimated using the four different GMPE's, it is generally of the same order of magnitude as the sample size uncertainty in the correlation coefficient itself.

Figure 5 also illustrates that for a given $I M_{i}$, the correlation with $C A V$ tends to be largest when that $I M_{i}$ is predicted using the CB08 GMPE. This observation is possibly a result of the fact that the CB08 GMPE uses the same functional form as the CB10 GMPE for CAV. However, it should also be noted that this observation is not overly significant, as indicated by the significant overlap between the boxplot distributions using the CB08 and other (i.e. BA08, CY08, AS08) GMPEs.

Figure 6 illustrates the empirical boostrapped distribution of the transformed correlation, $z$, between $C A V$ and $P G V$ (i.e. Figure 8b). Also shown is the normal distribution based on the point estimated mean and standard deviation of $z$, as well as the Lilliefors (Lilliefors 1967) goodness of fit bounds for the $10 \%$ significance level. The fact that the empirical distribution lies within these goodness of fit bounds signifies that the assumption of normality for $z$ cannot be rejected at the $10 \%$ significance level. The adequacy of the normal distribution (which was observed for all of the IM combinations examined) is useful when developing parametric correlation equations since it can be uniquely defined by its mean and standard deviation parameters.

Figure 7 illustrates the empirical correlation obtained between $C A V$ and $S A$ at various vibration periods. In Figure 7, the median and 90\% confidence interval of the correlation coefficient for a given GMPE are indicated by solid and dashed lines, respectively. Different line colours are used to represent the distribution of the correlation coefficient obtained using the four different GMPE's considered for each $I M_{i}$. It can be seen that the correlation between $C A V$ and $S A$ is largest (approximately 0.7) for short periods and reduces 
monotonically as the vibration period increases, in agreement with the observations in Figure 5. The results of Figure $\mathbf{7}$ demonstrate is that if a particular ground motion has a higher than expected $C A V$ value (based on the estimated distribution of $C A V$ for a given rupture scenario), then it is likely to be caused by a ground motion with larger than average spectral acceleration ordinates at all vibration frequencies, but most likely at high-to-moderate frequencies. That is, the correlations between the ground motion intensity measure residuals are relative to the predicted median values of the IM's and not to their absolute values.

It is worth noting that the size of the observed correlations in Figure 5 and Figure 7 have been discussed in a relative, and not absolute, sense. In an absolute sense, none of the observed correlations would be considered high in the author's opinion. For example, the correlation coefficient of $\rho_{\ln P G A, \operatorname{lnCAV}}=0.7$ means that given a specific value of $C A V$, the standard deviation of the conditional distribution of $P G A$ is reduced by only $29 \%$ from the unconditional distribution of PGA (Ang and Tang 2007). For DSI, in particular, $\left(\rho_{\text {lnDSI,lnCAV }}=0.565\right)$ the standard deviation of the conditional distribution is reduced by only $18 \%$. These relatively low correlations therefore indicate that the cumulative effects incorporated in the definition of $C A V$ can provide a significant amount of additional information about the severity of a ground motion in addition to the other, primarily amplitude-based, IMs examined here.

\section{PARAMETRIC MODELS FOR OBSERVED EMPIRICAL CORRELATIONS}

Given that the transformed correlation coefficient can be adequately represented by the normal distribution (i.e. Figure 6), it follows that the median value of the correlation coefficient, $\rho_{50}$, is equal to the inverse Fisher transformation of the mean of $z, \mu_{z}$, specifically:

$$
\rho_{50}=\left(\frac{e^{2 \mu_{z}}-1}{e^{2 \mu_{z}}+1}\right)=\tanh \left(\mu_{z}\right)
$$

Here parametric equations are developed for: (i) the median correlation coefficient, $\rho_{50}$, and (ii) the standard deviation of the transformed correlation coefficient, $\sigma_{z}$. By developing the equations in this manner, if an analyst wishes to neglect correlation coefficient uncertainty they can simply use $\rho_{50}$ directly. On the other hand, if one wants to explicitly account for correlation coefficient uncertainty then Equation (9) can be used to determine $\mu_{z}$ from the equation for $\rho_{50}$, and then with $\sigma_{z}$, the correlation coefficient for a given $x^{\text {th }}$ percentile can be obtained from: 


$$
\rho_{x}=\left(\frac{e^{2 z_{x}}-1}{e^{2 z_{x}}+1}\right)=\tanh \left(z_{x}\right)
$$

where $z_{x}$ is the $x^{\text {th }}$ percentile of a normal distribution with mean and standard deviation $\mu_{z}$ and $\sigma_{z}$, respectively (Ang and Tang 2007).

Table 1 provides the median correlation coefficient, $\rho_{50}$, and the standard deviation of the transformed correlation, $\sigma_{z}$, between $C A V$ and PGA, PGV, ASI, SI, and DSI. These values have been obtained directly from the mean and standard deviation of the $z$ values obtained from the aforementioned correlation analyses (i.e. Figure 5), after combining the results using the four GMPEs in a logic tree as previously discussed.

The correlation coefficients computed involving $C A V$ and $S A$ have 23 different values for the 23 vibration periods between $T=[0.01,10]$ for which $S A$ was computed for. In order to enable this correlation to be interpolated, and a smooth function of vibration period, a continuous parametric model was developed. The specific functional form used to represent the variation in the (median) correlation coefficient with vibration period is a piece-wise function with each piece-wise segment having the following functional form:

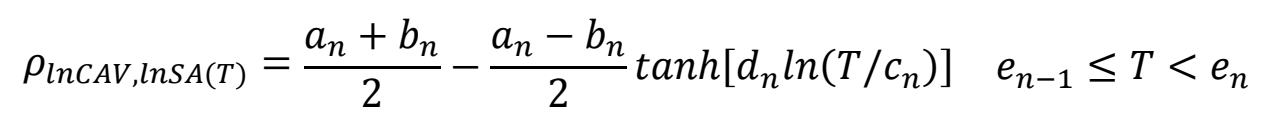

where tanh is the hyperbolic tangent function; $a_{n}, b_{n}, c_{n}$, and $d_{n}$ are empirical constants used to fit the observed empirical correlation coefficient for piece-wise segment $n$; and $e_{n}$ defines the period range for each of the piece-wise segments. Equation (11) was fit using least squares (Ang and Tang 1975) to minimize the misfit in the mean transformed $z$ value (i.e. to minimise the error between the Fisher transformation of Equation (11), which is normally distributed, and the empirical values of $\mu_{z}$ ). The parameters of each piece-wise segment were also constrained so that the parametric equation is a continuous piece-wise function of $T$. The obtained parametric equations were then plotted and compared with the empirical correlation values, and adjusted manually if necessary.

It was also found that there was a relatively simple variation of the standard deviation of the transformed correlation coefficient with vibration period. The variation was generally a decreasing standard deviation with increasing vibration period (with which the correlation coefficient also decreases), except for long periods, in which the effect of the reducing number of ground motions with appropriate useable periods caused an increase in the standard deviation. The specific parametric form of the transformed standard deviation model is: 


$$
\sigma_{z}= \begin{cases}0.055-0.0035 * \ln \left(\frac{T}{0.01}\right) & T<3.0 s \\ 0.055+0.0166 * \ln \left(\frac{T}{10}\right) & T \geq 3.0 s\end{cases}
$$

Table 2 provides the numerical values of the parameters in Equation (11) for the median correlation between $C A V$ with $S A(T)$, in which a total of three piece-wise segments were used. Figure 8a illustrates a comparison between the parametric correlation equations (i.e. Equations (11) and (12)) and the empirical correlation values obtained from the aforementioned analyses, while Figure 8b explicitly compares the empirical values of $\sigma_{z}$ with the parametric fit provided by Equation (12). In Figure 8a, the solid line represents the median correlation coefficient, $\rho_{50}$, while the dashed lines represent the $16^{\text {th }}$ and $84^{\text {th }}$ percentiles. It can be seen that the median correlation coefficient is well approximated by Equation (11). Both Figure 8a and Figure $8 \mathrm{~b}$ also illustrate that the simple function for the transformed standard deviation is adequate to represent the observed empirical values.

It was previously noted that the use of only a single GMPE for the prediction of $C A V$ (i.e. the CB10 model), as compared to the four different GMPE's used for predicting the other $I M_{i}$ 's considered, may result in an underestimation of the epistemic uncertainty in the correlation coefficients which contain $C A V$. Table 1 illustrates that the standard deviation in the transformed correlation coefficient between $C A V$ and PGA, PGV, ASI, SI, and DSI ranges from 0.043 to 0.055 , while Figure $8 \mathrm{~b}$ illustrates that for $C A V$ and $S A$ it ranges from 0.035 0.055 (neglecting the large increase for $S A(T=10 s)$ ). In comparison to the standard deviations obtained here, Bradley (2011) found that empirical correlations between $P G V$ and PGA, ASI, SI, and SA has standard deviations ranging from 0.036 to 0.046 (with the exception of long period $S A$ for which the standard deviation was up to 0.07), while those between DSI and PGA, PGV, ASI, SI and SA ranged from 0.045 to 0.062 (Bradley 2010a). Therefore it can be seen that the magnitude of the standard deviations in the transformed correlation coefficient involving CAV obtained here are of the same order (and often larger) than those obtained for other IM combinations.

\section{COMPARISON WITH THE CAV-PGA CORRELATION OF CAMPBELL AND BOZORGNIA (2010)}

Campbell and Bozorgnia (2010) use the PGA on bedrock to determine the non-linear site response terms in the CB10 CAV model. As such, when computing the standard deviation of $C A V$ one needs the correlation between $P G A$ (on rock) and $C A V$. It is worthy of note that in the CB10 GMPE the correlation between PGA and CAV is based on the intra-event residuals 
only, in comparison to the computation of the correlation between total residuals via inter- and intra-event correlations employed in this study. Figure 9 compares the parametric distribution of the CAV-PGA correlation $\left(\rho_{\ln C A V, \ln P G A}\right)$ model developed here, as given by the parameters in Table 1, with the tabulated correlation value of CB10. It can be seen that the correlation coefficient computed by CB08 is in agreement with the model developed in this study (i.e. the CB08 value differs from the median of the model developed here by less than $5 \%$ ). This is consistent with the fact that the correlation between total residuals is similar to that between intra-event residuals (e.g. Figure 4, and discussed in Bradley (2011)), and as previously mentioned the CB08 GMPE lead to a slightly larger correlation coefficient than the other three PGA GMPEs considered (i.e. Figure 5).

\section{USE OF CAV IN GROUND MOTION SELECTION}

As previously mentioned, the need for a ground motion intensity measure which accounts for cumulative effects lead EPRI (1988) to include $C A V$ as a parameter in determining the OBE exceedance in addition to response spectra. CAV can also be utilized explicitly in the determination of ground motions for seismic response analysis using the generalized conditional intensity measure (GCIM) approach (Bradley 2010b). Figure 10a illustrates the PGV seismic hazard curve computed using OpenSHA (Field et al. 2003) for an example rock site in California (Lat: 34.053, Long: $-118.243, V_{s, 30}=760 \mathrm{~m} / \mathrm{s}$ ) using the BA08 GMPE and the 1996 USGS earthquake rupture forecast (Frankel et al. 1996). For two particular exceedance probabilities of $10 \%$ and $1 \%$ in 50 years, Figure 10b illustrates the conditional distributions of $C A V$ given the corresponding $P G V$ values from the seismic hazard (Figure 10a), computed using the GCIM methodology with the CB10 CAV GMPE and the correlation equation for $C A V$ and $P G V$ developed here. It can be seen that the ratio of the two $P G V$ values for these exceedance probabilities is $46.9 / 25.7=1.82$, while the ratio of the median values of the conditional $C A V$ distributions is $1.00 / 0.69=1.44$. That is, while the $P G V$ value of ground motions at the two hazard levels increases by $82 \%$, the median conditional $C A V$ value increases by only $44 \%$. This difference illustrates the changing nature of the time history of the ground motions which pose a hazard to the site. Given that both $P G V$ and $C A V$ scale linearly with the amplitude scale factor (i.e. amplitude scaling a ground motions' $P G V$ by $82 \%$ will also scale its $C A V$ by $82 \%$ ), the result of Figure 10b clearly shows that care is needed to avoid excessive amplitude-scaling of ground motions which will result in incorrect distributions for intensity measures other than that upon which the scaling is based. Clearly these features of the changing nature of the expected ground motions, at different exceedance 
probabilities in a seismic hazard analysis, will influence seismic response analysis and therefore should be accounted for by selecting ground motion records which provide the correct conditional distribution of intensity measures (such as CAV) for which a seismic response analysis is sensitive (as elaborated upon by Bradley (2010b)).

\section{CONCLUSIONS}

This manuscript has examined and developed parametric equations for the correlation (for a given earthquake rupture) between cumulative absolute velocity (CAV) and other common ground motion intensity measures (IMs), namely, peak ground acceleration (PGA), peak ground velocity (PGV), 5\% damped pseudo spectral acceleration $(S A)$, acceleration spectrum intensity ( $A S I)$, spectrum intensity ( $S I)$, and displacement spectrum intensity ( $D S I)$ from active shallow crustal earthquakes. Because of the cumulative nature of $C A V$ it was found that, in an absolute sense, strong correlations (i.e. $\rho>0.9$ ) were not observed with any of the examined IMs, which are primarily amplitude-based in nature. In a relative sense, the largest correlations were observed for short period IMs such as PGA and ASI (0.70 and 0.703, respectively), followed by moderate periods IMs such as PGV and SI (0.691 and 0.681, respectively).

Based on the developed correlation equations it was illustrated how distributions of $C A V$ can be developed for different exceedance probabilities from seismic hazard analysis. The observation that $C A V$ scales differently from amplitude-based intensity measures clearly illustrates the changing nature of the expected ground motions for different seismic hazard exceedance probabilities, which should be accounted for in ground motion selection based on such distributions.

\section{REFERENCES:}

Abrahamson, N. A., Atkinson, G. M., Boore, D. M., Bozorgnia, Y., Campbell, K. W., Chiou, B., Idriss, I. M., Silva, W. J., Youngs, R. R., 2008. Comparisons of the NGA Ground-Motion Relations, Earthquake Spectra, 24, 45-66.

Abrahamson, N. A., Silva, W. J., 2008. Summary of the Abrahamson \& Silva NGA ground motion relations, Earthquake Spectra, 24, 67-97.

Abrahamson, N. A., Youngs, R. R., 1992. A stable algorithm for regression analyses using the random effects model, Bulletin of the Seismological Society of America, 82, 505-510.

Akkar, S., Bommer, J. J., 2007. Empirical prediction equations for peak ground velocity derived from strong-motion records from Europe and the Middle East, Bulletin of the Seismological Society of America, 97, 511-530.

Ang, A. H. S., Tang, W. H., 1975. Probability concepts in engineering planning and design John Wiley \& Sons, Inc. p. 406. 
Ang, A. H. S., Tang, W. H., 2007. Probability concepts in engineering: Emphasis on applications in civil and environmental engineering. John Wiley \& Sons.

Boore, D. M., Atkinson, G. M., 2008. Ground-motion prediction equations for the average horizontal component of PGA, PGV, and 5\%-damped PSA at spectral periods between $0.01 \mathrm{~s}$ and 10.0s, Earthquake Spectra, 24, 99-138.

Bradley, B. A., 2009. Site specific and spatially distributed prediction of acceleration spectrum intensity, Bulletin of the Seismological Society of America, 100, 792-801.

Bradley, B. A., 2010a. Displacement spectrum intensity and its use in ground motion selection, Soil Dynamics and Earthquake Engineering.

Bradley, B. A., 2010b. A generalized conditional intensity measure approach and holistic ground motion selection, Earthquake Engineering and Structural Dynamics, 39, 1321-1342.

Bradley, B. A., 2011. Empirical correlations between peak ground velocity and spectrumbased intensity measures, Earthquake Spectra.

Bradley, B. A., Cubrinovski, M., Dhakal, R. P., MacRae, G. A., 2009a. Intensity measures for the seismic response of pile foundations, Soil Dynamics and Earthquake Engineering, 29, 1046-1058.

Bradley, B. A., Cubrinovski, M., MacRae, G. A., Dhakal, R. P., 2009b. Ground motion prediction equation for spectrum intensity from spectral acceleration relationships, Bulletin of the Seismological Society of America, 99, 277-285.

Campbell, K. W., Bozorgnia, Y., 2008. NGA Ground Motion Model for the Geometric Mean Horizontal Component of PGA, PGV, PGD and 5\% Damped Linear Elastic Response Spectra for Periods Ranging from 0.01 to $10 \mathrm{~s}$ Earthquake Spectra, 24, 139-171.

Campbell, K. W., Bozorgnia, Y., 2010. A Ground Motion Prediction Equation for the Horizontal Component of Cumulative Absolute Velocity (CAV) Based on the PEER-NGA Strong Motion Database, Earthquake Spectra, 26, 635-650.

Chen, Y.-H., Tsai, C.-C. P., 2002. A New Method for Estimation of the Attenuation Relationship with Variance Components, Bulletin of the Seismological Society of America, 92, 1984-1991.

Chiou, B., Darragh, R., Gregor, N., Silva, W. J., 2008. NGA project strong-motion database, Earthquake Spectra, 24, 23-44.

Chiou, B., Youngs, R. R., 2006. Chiou and Youngs PEER-NGA Empirical Ground Motion Model for the Average Horizontal Component of Peak Acceleration and Pseudo-Spectral Acceleration for Spectral Periods of 0.01 to 10 Seconds, 219pp.

Chiou, B. S. J., Youngs, R. R., 2008. An NGA Model for the average horizontal component of peak ground motion and response spectra, Earthquake Spectra, 24, 173-215.

Danciu, L., Tselentis, G. A., 2007. Engineering ground motion attenuation relationships for Greece, Bulletin of the Seismological Society of America, 97, 162-183.

EPRI, 1988. A Criterion for Determining Exceedance of the Operating Basis Earthquake, EPRI NP-5930, Palo Alto, California. 330pp.

EPRI, 2006. Program on Technology Innovation: Use of Cumulative Absolute Velocity (CAV) in Determining Effects of Small Magnitude Earthquakes on Seismic Hazard Analyses, 1014099, Palo Alto, California. 190pp.

Field, E. H., Jordan, T. H., Cornell, C. A., 2003. OpenSHA: A developing communitymodelling environment for seismic hazard analysis, Seismological Research Letters, 74, 406419.

Frankel, A., Mueller, C., Barnhard, T., Perkins, D., Leyendecker, E., Dickman, N., Hanson, S., Hopper, M., 1996. National Seismic Hazard Maps-Documentation, U.S. Geological Survey, Open-File Report 96-532, U.S. Geological Survey, 110pp. 
Housner, G. W., 1952. Spectrum intensities of strong-motion earthquakes, in Symposium on earthquakes and blast effects on structures. Earthquake Engineering Research Institute: Los Angeles, CA.

Jayaram, N., Baker, J. W., 2010. Considering spatial correlation in mixed-effects regression, and impact on ground-motion models, Bulletin of the Seismological Society of America.

Koliopoulos, P. K., Margaris, B. N., Klimis, N. S., 1998. Duration and energy characteristics of Greek strong motion records, Journal of Earthquake Engineering, 2, 391 - 417.

Kramer, S. L., Mitchell, R. A., 2006. Ground motion intensity measures for liquefaction hazard evaluation, Earthquake Spectra, 22, 413-438.

Kulkarni, R. B., Youngs, R. R., Coppersmith, K. J., 1984. Assessment of confidence intervals for results of seismic hazard analysis, in 8th World Conference on Earthquake Engineering,: San Francisco, CA. p. 263-270.

Lilliefors, H., 1967. On the Kolmorogov-Smirnov test for normality with mean and variance unknown, Journal of the American Statistical Association, 62, 399-402.

Peruš, I., Fajfar, P., 2010. Ground-motion prediction by a non-parametric approach, Earthquake Engineering \& Structural Dynamics, 39, 1395-1416.

Power, M., Chiou, B., Abrahamson, N. A., Bozorgnia, Y., Shantz, T., Roblee, C., 2008. An overview of the NGA project, Earthquake Spectra, 24, 3-21.

Scasserra, G., Stewart, J. P., Bazzurro, P., Lanzo, G., Mollaioli, F., 2009. A Comparison of NGA Ground-Motion Prediction Equations to Italian Data, Bulletin of the Seismological Society of America, 99, 2961-2978.

Shoja-Taheri, J., Naserieh, S., Hadi, G., 2010. A Test of the Applicability of NGA Models to the Strong Ground-Motion Data in the Iranian Plateau, Journal of Earthquake Engineering, 14, 278 - 292.

Stafford, P. J., Strasser, F. O., Bommer, J. J., 2008. An evaluation of the applicability of the NGA models to ground-motion prediction in the Euro-Mediterranean region, Bulletin of Earthquake Engineering, 6, 149-177.

Von Thun, J., Roehm, L., Scott, G., Wilson, J., 1988. Earthquake ground motions for design and analysis of dams, in Earthquake Engineering and Soil Dynamics II - Recent Advances in Ground-Motion Evaluation, Geotechnical Special Publication. p. 463-481. 
Table 1: Proposed median correlation, $\rho_{50}$, and the standard deviation of the transformed correlation, $\sigma_{z}$ (shown in brackets) of DSI with PGA, PGV, ASI, and SI.

\begin{tabular}{|c|c|c|c|c|c|}
\hline $\begin{array}{c}\rho_{50} \\
\left(\sigma_{z}\right)\end{array}$ & $P G A$ & $P G V$ & $A S I$ & $S I$ & $D S I$ \\
\hline$C A V$ & 0.700 & 0.691 & $\begin{array}{c}0.703 \\
(0.052)\end{array}$ & $\begin{array}{c}0.681 \\
(0.044)\end{array}$ & $\begin{array}{c}0.565 \\
(0.043)\end{array}$ \\
\hline
\end{tabular}

Table 2: Parameters in Equation (11) defining the piece-wise variation of the median correlation between $C A V$ and $S A(T), \rho_{\ln C A V, \ln S A(T)}$.

\begin{tabular}{|c|c|c|c|c|c|}
\hline$n$ & $e_{n}$ & $a_{n}$ & $b_{n}$ & $c_{n}$ & $d_{n}$ \\
\hline 0 & 0.01 & - & - & - & - \\
\hline 1 & 0.20 & 0.70 & 0.635 & 0.043 & 2.5 \\
\hline 2 & 3.0 & 0.635 & 0.525 & 0.95 & 3.0 \\
\hline 3 & 10.0 & 0.525 & 0.39 & 6.2 & 4.0 \\
\hline
\end{tabular}



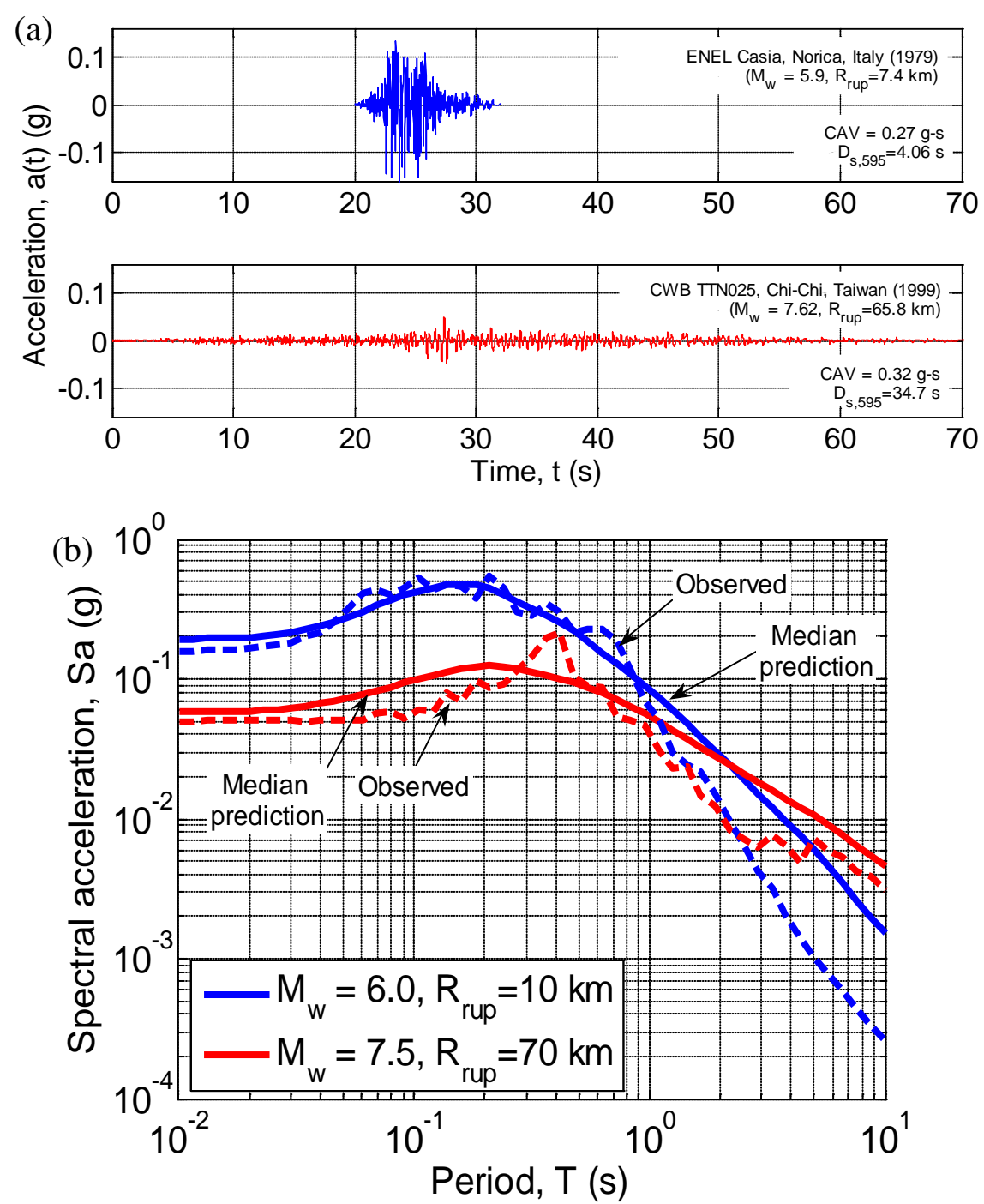

Figure 1: Comparison of two ground motions with similar $C A V$ values: (a) time histories; (b) predicted median and observed pseudo acceleration response spectra. 


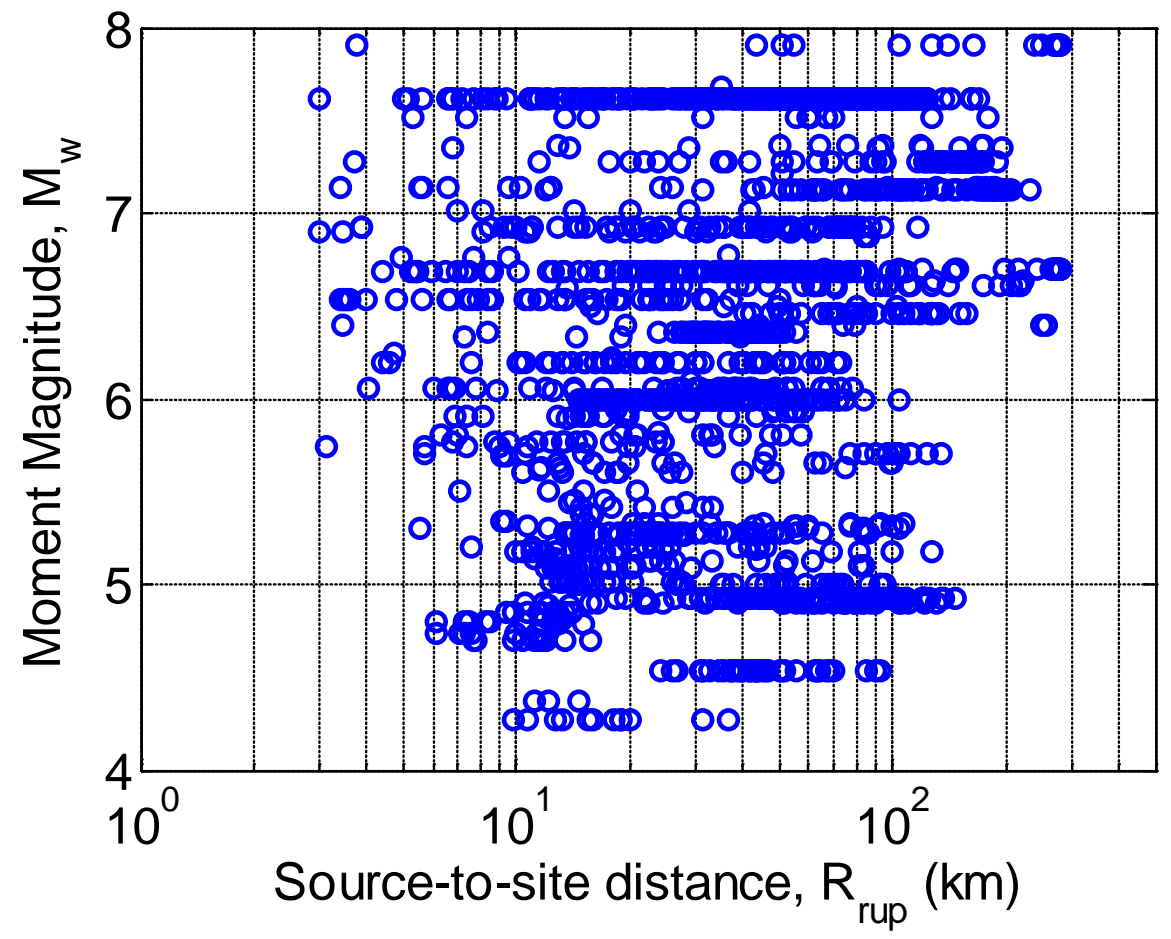

Figure 2: Moment magnitude and source-to-site distance distribution of the ground motion dataset used. 

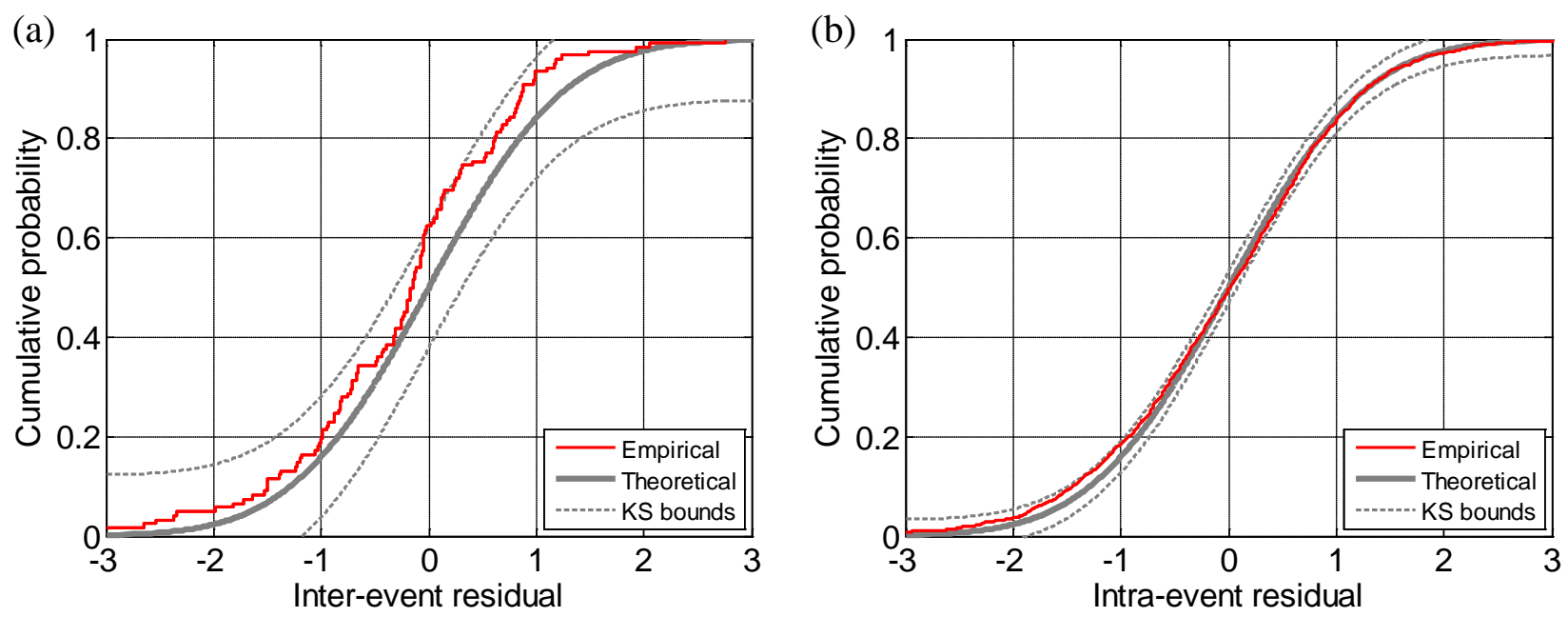

Figure 3: Distributions of (a) normalized inter-event; and (b) normalized intra-event residuals obtained using the Campbell and Bozorgnia (2010) (CB10) CAV model and the ground motion dataset used in this study. 
(a)

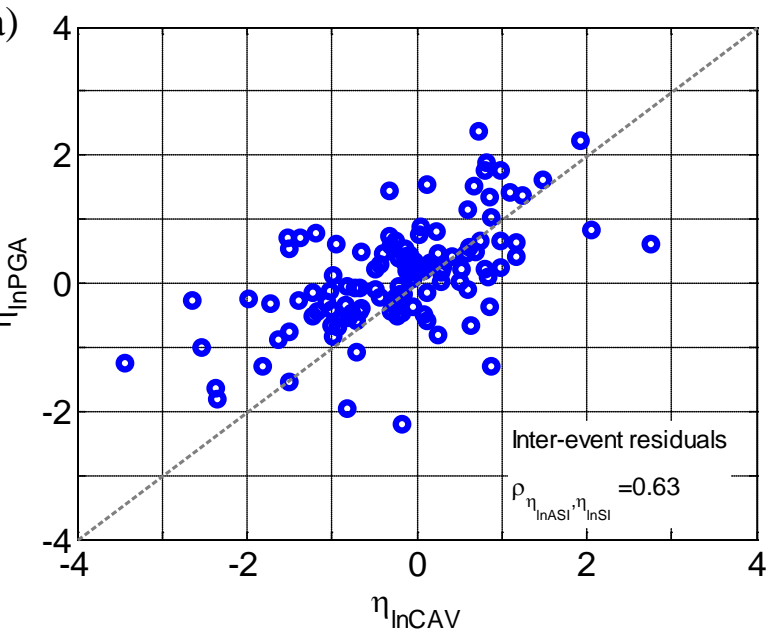

(b)

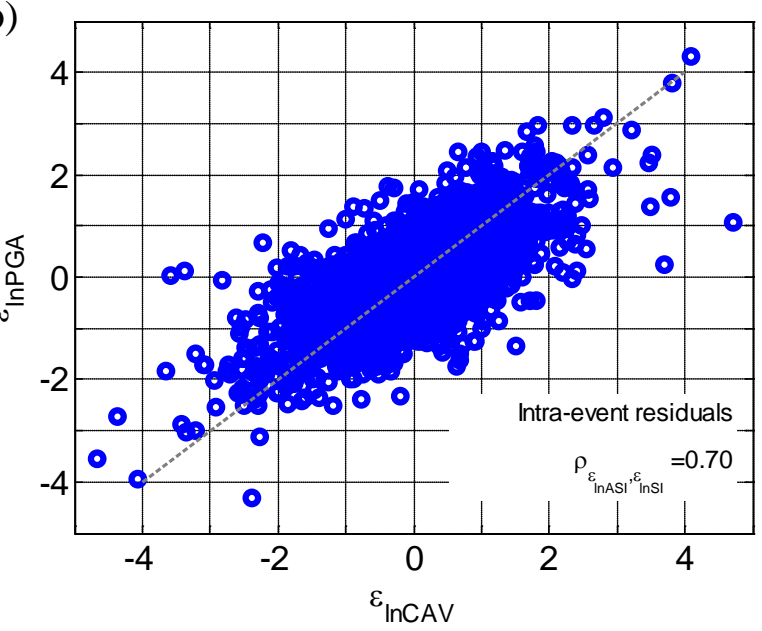

Figure 4: Examples of the correlation obtained between $C A V$ and $P G A$ for a single bootstrap sample and using the BA08 PGA GMPE: (a) inter-event residuals; and (b) intra-event residuals. The correlation between total residuals for this example via Equation (6) is 0.687. 

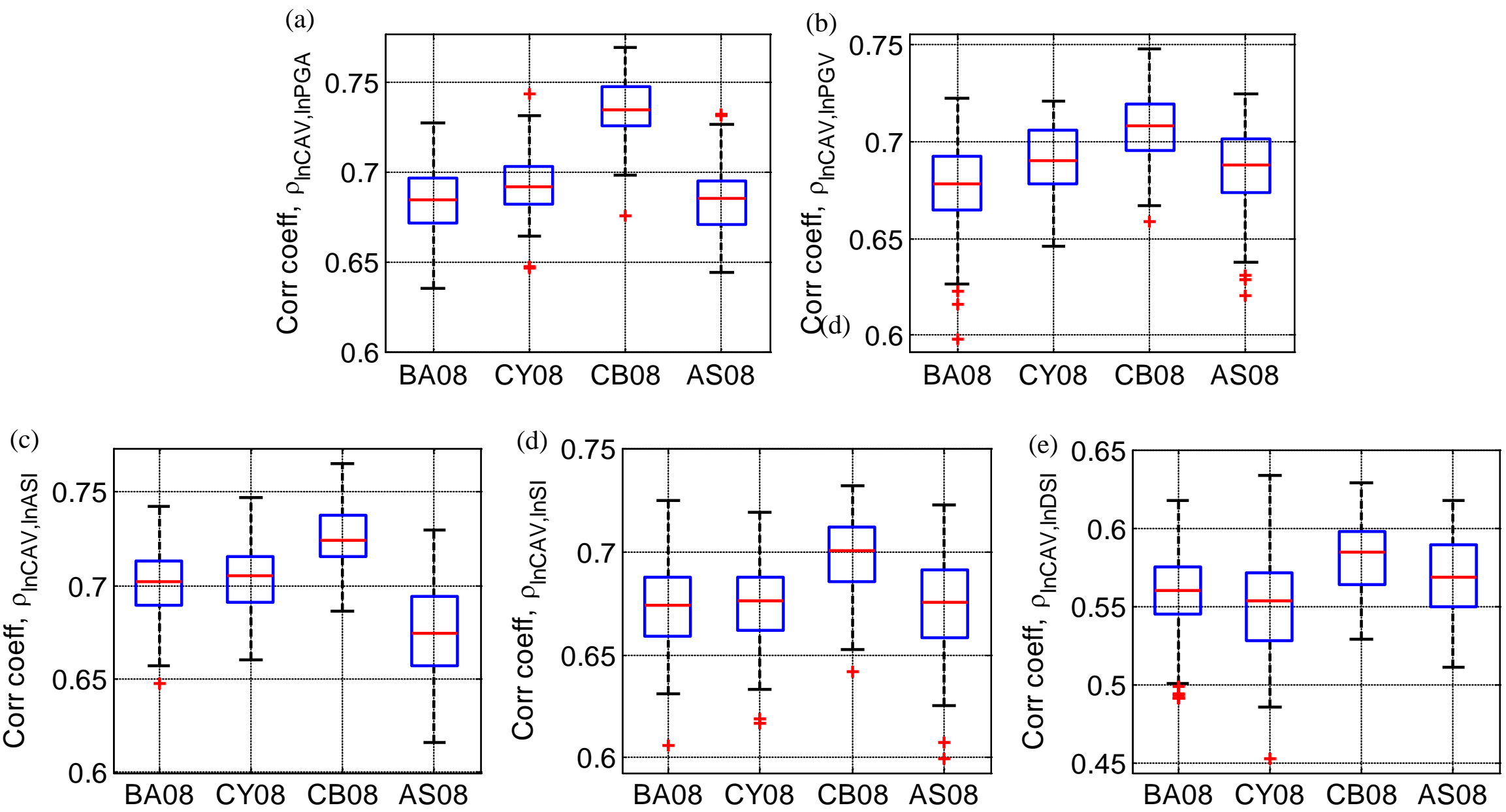

Figure 5: Correlation coefficients obtained between $C A V$ and: (a) PGA; (b) PGV; (c) ASI; (d) $S I$ and (e) DSI. In each figure, box plots represent the uncertainty due to the finite sample size, while the different box plots illustrate the uncertainty due to the selected GMPE. 


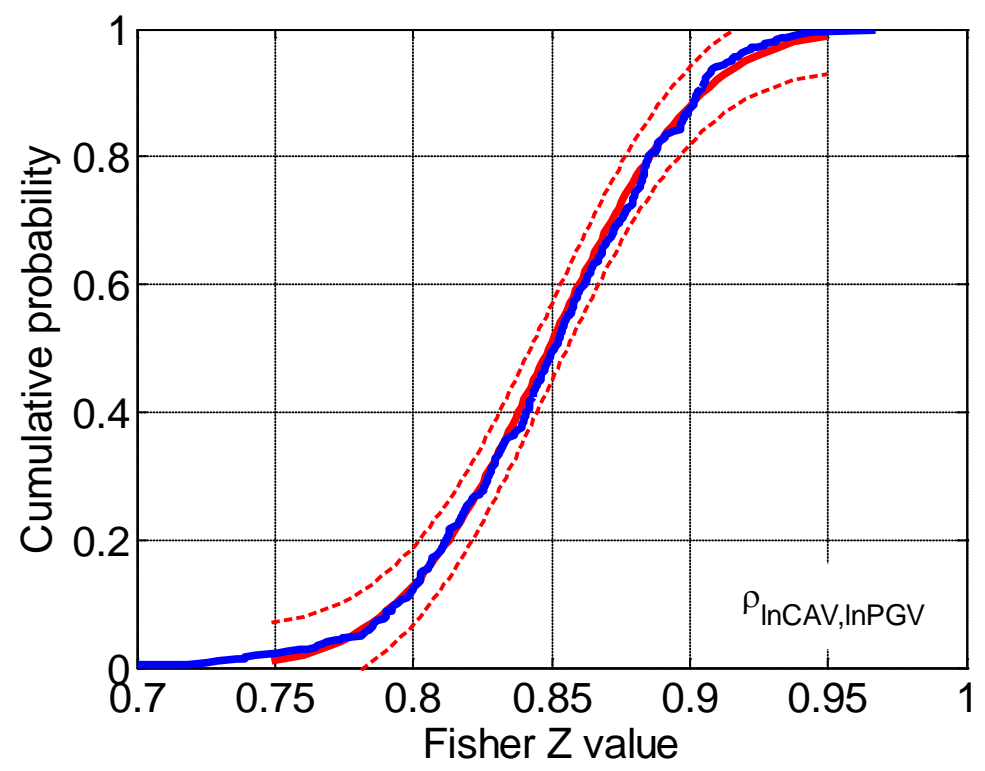

Figure 6: Illustration of the adequacy of the approximation that $z$ has a normal distribution due to sample size and GMPE uncertainty for the correlation between $C A V$ and $P G V$. 


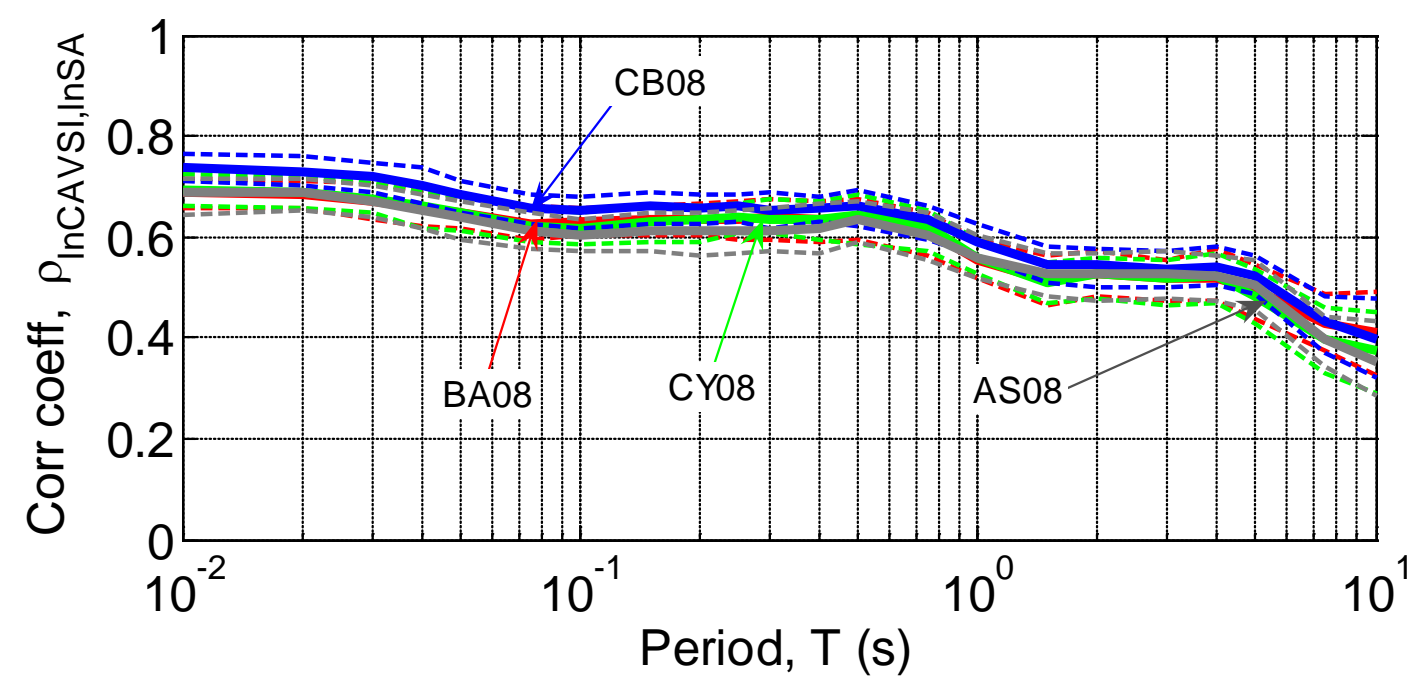

Figure 7: Correlation coefficients obtained between $C A V$ and $S A$. The solid and dashed lines represent the median and $90 \%$ confidence interval due to finite sample size, while the different coloured lines illustrate the uncertainty due to the selected GMPE. 

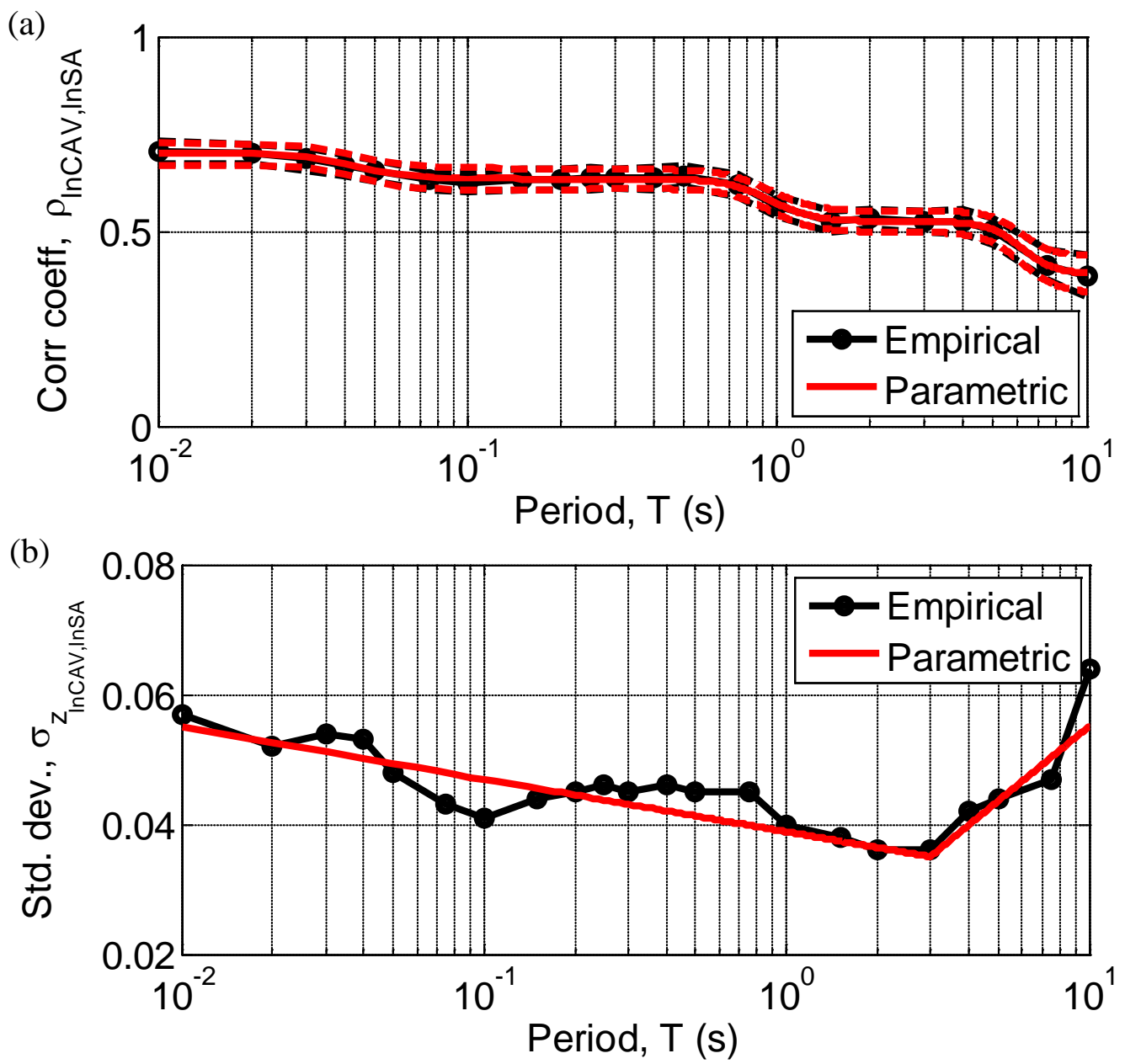

Figure 8: Comparisons of the empirical correlations and parametric fit of $C A V$ and $S A$ : (a) median, $16^{\text {th }}$ and $84^{\text {th }}$ percentiles; and (b) standard deviation. 


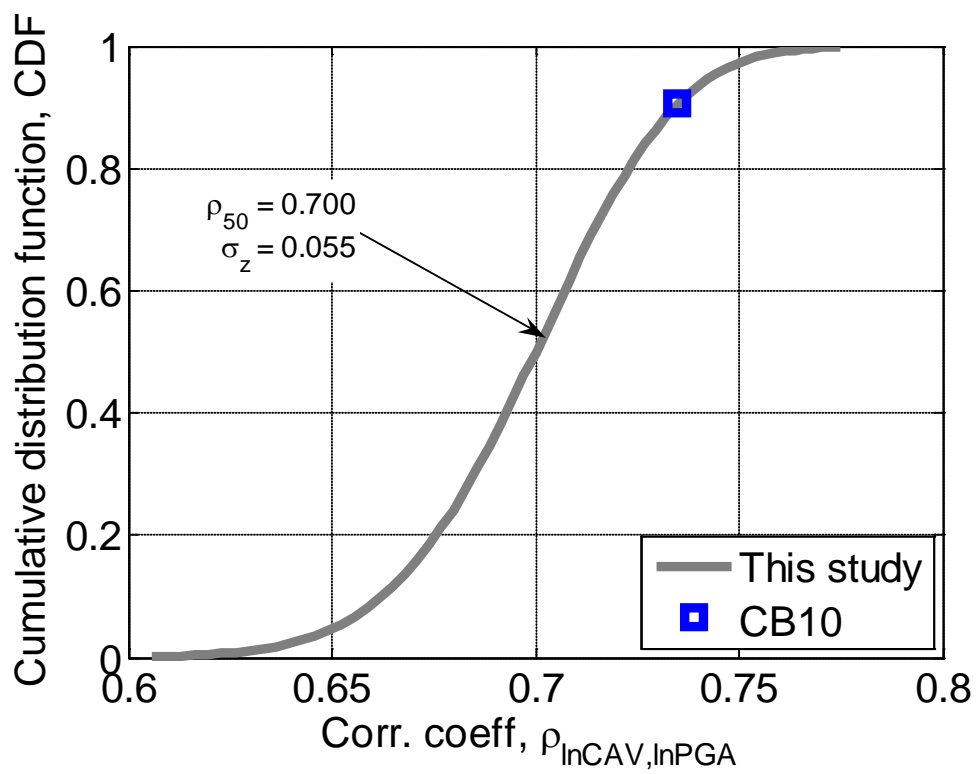

Figure 9: Comparison of the parametric correlation model for $C A V$ and PGA developed in this study with that of Campbell and Bozorgnia (Campbell and Bozorgnia 2010) (CB10). 

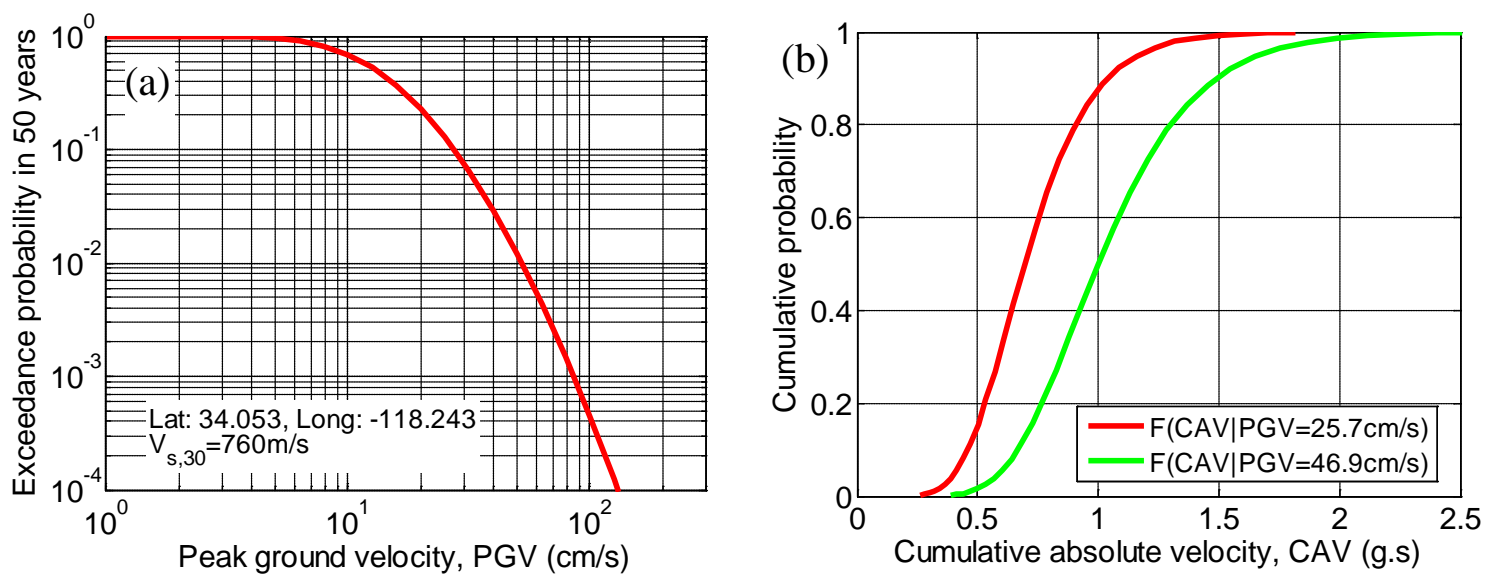

Figure 10: (a) Seismic hazard curve for peak ground velocity; and (b) conditional distributions of $C A V$ given $P G V$ corresponding to $10 \%$ and $1 \%$ probabilities of exceedance in 50 years. 